\section{Bem-estar subjetivo em praticantes e não praticantes de meditação}

\author{
Débora Noemi Hernández \\ Gustavo Henrique Martins
}

\section{RESUMO}

O Bem-Estar Subjetivo (BES) é composto por Afetos Positivos (AP), Negativos (AN) e Satisfação com a Vida (SV), que se referem aos componentes afetivos e cognitivos. As práticas meditativas, incorporadas às práticas integrativas aprovadas pelo SUS, são utilizadas para o relaxamento, incluindo alívio emocional e mental, e redução do estresse. Assim, este estudo procurou identificar relações entre os escores do BES e as práticas de meditação, frequência e anos de prática. Esta pesquisa foi composta por praticantes ( $\mathrm{n}=$ $166)$ e não praticantes $(n=139)$, os quais responderam a Escala de Bem-Estar Subjetivo. Obteve-se como resultado três modelos preditivos, indicando que os praticantes de meditação obtiveram maiores índices em AP e SV, bem como menores índices em AN. Também se observaram efeitos significativos e positivos da frequência e anos de prática sobre o BES. Em suma, os resultados obtidos podem indicar haver evidências de que as práticas de meditação possuem efeitos sobre o BES dos indivíduos.

Palavras-chave: medicina alternativa; saúde mental; emoções; felicidade.

\section{ABSTRACT}

\section{Subjective well-being in meditation practitioners and non-practitioners}

Subjective Well-Being (SWB) is composed of Positive Affects (PA), Negative Affects (NA) and Life Satisfaction (LF), which refers to affective and cognitive components. Meditative practices were incorporated into integrative practices approved by the public health department, and are used for relaxation, including emotional and mental relief, and stress reduction. Thus, this study sought to identify relationships between BES scores and practices of meditation, frequency and years of practice. This research was composed by practitioners $(N=166)$ and non-practitioners $(N=139)$, who responded to the Subjective Well-Being Scale. As a result, three predictive models were obtained, indicating that meditation practitioners presented higher rates of PA and LF, as well as lower rates of NA. Significant and positive effects of frequency and years of practice on BES were also observed. In short, the results obtained may indicate that there is evidence that meditation practices have effects on the SWB of individuals.

Keywords: complementary therapies; mental health; emotions; happiness.

Muitos se questionam sobre o que é felicidade? Não é uma pergunta fácil de responder, mas na perspectiva hedônica adotada por Diener (2000), felicidade pode ser denominada como Bem-Estar Subjetivo (BES). Esse conceito concede ao sujeito o direito de decidir se é ou não feliz. O BES pode ser compreendido por meio de dois componentes, o afetivo

\section{Sobre os Autores}

D.N.H

http://orcid.org/0000-0003-16893272

Universidade São Francisco,

Campinas - SP

debi_hernandez@hotmail.com

G.H.M

http://orcid.org/0000-0002-51252553

Universidade São Francisco,

Campinas - SP

gustavoh.martins95@gmail.com

\section{Direitos Autorais}

Este é um artigo de acesso berto e pode ser reproduzido livremente, distribuído, transmitido ou modificado, por qualquer pessoa desde que usado sem fins comerciais. O trabalho é disponibilizado sob a licença Creative Commons CC-BY-NC. 
e o cognitivo. O componente cognitivo é representado pela Satisfação com a Vida, a qual consiste em um julgamento cognitivo de algum aspecto da vida do sujeito, um processo de juízo e avaliação geral da própria vida, ou uma avaliação sobre a vida conforme um critério próprio (Emmons, 1986; Keyes et al., 2002; Seligman, 2011; Shin \& Johnson, 1978). O julgamento da satisfação depende de uma comparação entre as circunstâncias de vida do sujeito e um padrão por ele escolhido (Albuquerque \& Tróccoli, 2004).

Os afetos são a resposta imediata que uma pessoa tem a um estímulo ou acontecimento, estes geralmente baseiam-se em uma sensação de excitação, a qual permite avaliar o evento como prazeroso ou doloroso para o sujeito (Snyder \& Lopez, 2009). Entende-se que os afetos interferem ativamente na maneira como o sujeito vê a própria vida e as pessoas ao seu redor. Dessa forma, as pessoas que possuem maior prevalência de afetos positivos tendem a sentir mais prazer em suas atividades cotidianas, no entanto, aqueles que possuem mais afetos negativos, a tendência é oposta, sendo que seu olhar para o cotidiano tenderá a ser negativo e triste (Watson et al., 1988).

O componente afetivo do BES é divido em Afetos Positivos e Afetos Negativos. Entende-se que os Afetos Positivos dizem respeito ao quanto uma pessoa está sentindo-se entusiasmada, ativa, alerta, jovial, autoconfiante e atenciosa. Refere-se a um contentamento hedônico puro, experienciado em determinado momento como um estado de atenção e de atividade. Seria ainda um sentimento transitório de prazer ativo, referindo-se à descrição de um estado emocional, e não um julgamento cognitivo (Watson et al., 1988). Ao passo que os Afetos Negativos se referem à dimensão geral da angústia e insatisfação, um desconforto, incluindo estados de humor aversivos, como raiva, culpa, desgosto e medo. Os Afetos Negativos dizem respeito a um estado de distração incluindo emoções desagradáveis, porém também transitório. Portanto, um alto BES significa altos índices de Afetos Positivos e Satisfação com a Vida e baixos Afetos Negativos (Diener, 2000).

A forma mais utilizada para mensuração do BES é o uso do autorrelato, no qual o sujeito julga a satisfação que possui em relação à sua própria vida e à frequência de afetos, no que se refere a prazer e desprazer na mesma. Considera-se que essa forma de avaliação é adequada, visto que apenas o sujeito pode experimentar os prazeres ou desprazeres e julgar o quão satisfeito está com sua vida (Albuquerque \& Tróccoli, 2004; Diener et al., 2003; Larsen et al., 1985). No Brasil, um dos instrumentos utilizados para essa avaliação é a Escala de Bem-Estar Subjetivo (EBES; Albuquerque \& Tróccoli, 2004; Pires et al., 2015; Pureza et al., 2012). A EBES foi utilizada no Brasil para avaliação do BES em diferentes contextos, tais como estudos sobre depressão, personalidade e regulação emocional, conjugalidade dos pais, estado civil (Baptista et al., 2016; Ribeiro et al., 2018; ScorsoliniComin et al., 2016; Scorsolini-Comin et al., 2015).

O BES tem impactos a curto e a longo prazo na vida das pessoas, sendo essa uma variável importante para a saúde mental dos indivíduos (Lyubomirsky et al., 2005; Walsh et al., 2018). Assim, práticas que visem aumentar os níveis de BES tem impactos positivos para o sucesso dos indivíduos bem como para a sociedade como um todo. O BES está relacionado à prevenção primária, que se refere ao trabalho de identificação de fatores negativos antes que algo ruim aconteça, procurando evitar o adoecimento (Duckworth et al., 2005; Snyder \& Lopez, 2009). A potencialização primária diz respeito a tornar a "vida boa", para isso, alguns autores da psicologia positiva sugerem a prática de meditação, dado que a apreciação do presente, do ambiente interno e externo, permite que o sujeito tenha uma experiência de paz, calma e prazer (Shapiro et al., 2002).

De forma geral, de acordo com Mendoza (2017), a meditação é reconhecida como uma forma de treinar a mente, assim como o exercício físico treina o corpo, sendo assim, meditar quer dizer manter a concentração em um som, objeto, momento, ou em si mesmo, para aumentar a atenção no momento presente. Existem diversas técnicas, provenientes de diferentes culturas e tradições, porém essa prática, como um todo, é conhecida por promover profundo relaxamento e redução do estresse, calma emocional e mental, expansão de consciência, melhorias pessoais e crescimento espiritual. Nikhra (2016) também indica que a meditação leva os indivíduos a tomarem um caminho mais positivo, por transformar a sua forma de pensamento e de perceber as situações.

Dessa forma, entendendo que o BES é sinônimo de felicidade e que, conforme indicado acima, a meditação é reconhecida por promover alívio dos fatores que poderiam impedir a percepção do mesmo, entende-se que existe a necessidade de avaliar a veracidade científica de tal interfase teórica. Ainda pensando que a utilização desse tipo de prática tem aumentado após a aprovação das práticas integrativas no SUS, porém destacando que ainda não existem muitas pesquisas cientificas nacionais sobre os efeitos das mesmas.

Em janeiro de 2017, o Sistema Único de Saúde 


\section{HET INTERACÃO EM
PSICOLOGIA}

(SUS) incluiu algumas práticas integrativas aos serviços fornecidos, dentre eles, a meditação, por meio da Portaria $N^{\circ} 145 / 2017$. Isso é decorrente da Política Nacional de Práticas Integrativas e Complementares (PNPIC), criada em 2006. Destaca-se ainda que as práticas integrativas também são reconhecidas pela Organização Mundial da Saúde. As práticas integrativas e complementares se referem às práticas não hegemônicas de produção de cuidado, isto é, práticas não convencionais de terapia como acupuntura, homeopatia, plantas medicinais/ fitoterápicas, medicina natural, medicina antroposófica, medicina tradicional chinesa, entre outras (Ministério da Saúde, 2019; Moebus \& Merhy, 2017; Portaria 145/2017; World Health Organization, 2013).

Nesse sentido, diversas pesquisas foram realizadas com o intuito de investigar a relação entre a prática de meditação e o BES. Goyal et al. (2014) buscaram identificar os efeitos da meditação Mindfulness nos Afetos Positivos e Negativos por meio de uma metanálise, que incluiu 47 estudos. Segundo os resultados obtidos, não foram encontradas evidências de alterações significativas nos escores de Afetos Positivos nos praticantes dessa meditação. Por outro lado, foram observados efeitos positivos nos escores de Afetos Negativos, com a diminuição dos mesmos. Outra metanálise testou os efeitos da Kindness-Based Meditation (Meditação da Bondade) sobre a saúde e bem-estar. As análises feitas, a partir de 22 estudos, observaram uma diminuição de sintomas depressivos e aumento dos níveis de Afetos Positivos e autocompaixão (Galante et al., 2014).

Shonin et al., (2014) realizaram sua pesquisa com base na Meditation Awareness Training, de origem Budista. Os autores procuraram identificar os efeitos dessa meditação por meio do treinamento de 10 participantes durante oito semanas. Dentre os resultados da pesquisa, verificamse os efeitos positivos no BES dos participantes dessa meditação. No estudo de Bach e Guse (2014), por sua vez, foi realizado um treinamento de Meditação Contemplativa. Durante a pesquisa, os participantes foram divididos em dois grupos, controle $(n=25)$ e experimental $(n=26)$. Os resultados apontaram que os participantes do grupo experimental apresentaram uma diminuição significativa dos Afetos Negativos, e um aumento significativo em Satisfação com a Vida em comparação com o grupo controle. Não houve alterações significativas nos Afetos Positivos.

Tarrant et al., (2016) procuraram mostrar o impacto da prática da Meditação dos Corações Gêmeos em funções psicológicas. Houve uma avaliação antes e depois dos participantes serem submetidos a 30 minutos
Débora Noemi Hernández, Gusta vo Henrique Martins

de Meditação dos Corações Gêmeos. Os participantes foram divididos em dois grupos, pessoas experientes ( $n=12)$, com mais de 1000 horas de prática, e pessoas novatas $(n=12)$, que nunca tinham praticado nenhum tipo de meditação. Dentre os resultados obtidos, notaramse resultados imediatos nos participantes novatos, com uma diminuição significativa da ansiedade e melhora na função cognitiva. Além disso, os participantes novatos obtiveram aumento significativo dos níveis de felicidade imediatamente após a meditação. Os resultados também sugerem que os participantes experientes têm níveis mais elevados de autoeficácia, felicidade e menores níveis de ansiedade.

No Brasil foram recuperados apenas três estudos empíricos que visaram relacionar a prática de meditação e o BES. Menezes e Dell'Aglio (2009) investigaram como os praticantes da meditação passiva (sentada e silenciosa) percebiam os benefícios dessa prática. Foi realizada uma análise de conteúdo das respostas dos 105 participantes à questão aberta sobre como a meditação se refletia em suas vidas. As respostas foram categorizadas em cinco dimensões: cognitiva (autoconhecimento, atenção e concentração, objetividade, centramento e êxito na utilização de estratégias de coping); emocional (tranquilidade, paz, equilíbrio emocional, bem-estar, autoestima, segurança e redução do estresse e ansiedade); física (relaxamento do corpo e melhor qualidade do sono e da saúde em geral); social (maior aceitação dos outros e sensação de pertencimento a um grupo); e espiritual (sentimento de conexão com algo maior, aproximação de Deus e equilíbrio espiritual). Por fim, as autoras discutem que a prática de meditação pode se refletir de diversas formas, com predomínio de benefícios cognitivos e emocionais.

Outro estudo publicado pelas mesmas autoras buscou investigar a relação entre número de meses, frequência diária e semanal, e duração de cada prática de meditação sobre o bem-estar psicológico. A amostra foi composta por 142 praticantes da meditação passiva. Como resultados, as autoras relatam que o número de meses e a frequência semanal da prática de meditação relacionaram-se positivamente com o bem-estar psicológico. Além disso, foi visto que os traços de personalidade Conscienciosidade, Extroversão e Neuroticismo mediaram essa relação (Menezes \& Dell'Aglio, 2010). Por fim, Menezes e Bizarro (2015) buscaram avaliar os efeitos de cinco dias de meditação sobre os Afetos Positivos, Afetos Negativos, Ansiedade Estado e Traço e Atenção Concentrada. A amostra do estudo foi composta por 14 participantes do grupo experimental e 19 do grupo controle. Os resultados 


\section{HZZ INTERACÃO EM
PSICOLOGIA}

indicaram diminuição dos Afetos Negativos e Ansiedade Traço e aumento da Atenção Concentrada do grupo experimental em comparação com o grupo controle.

Além da relação entre meditação e BES apresentada anteriormente, alguns autores apontam que características sociodemográficas podem ter efeitos sobre o BES, como sexo (Batz \& Tay, 2018; Freire \& Tavares, 2011; Noronha et al., 2016; Silva et al., 2010; Zanon \& Hutz 2009), idade (Carochinho, 2018; Cunha et al., 2017; Otta \& Fiquer 2004) e escolaridade (Cachioni et al., 2017). Entende-se que participantes dessas práticas não apenas realizam uma técnica, mas representam um estilo de vida. Esse estilo de vida pode influenciar os diversos aspectos da vida, dependendo da profundidade com a qual o sujeito está comprometido com este (Gaiswinker et al., 2015). Dessa forma, é possível compreender que o impacto das práticas de meditação no BES do sujeito depende do envolvimento nas mesmas (Bussing et al., 2012).

Diante do cenário apresentado, o objetivo da presente pesquisa foi investigar as diferenças no BES entre praticantes e não praticantes de meditação, controlando os efeitos da idade, sexo e escolaridade. Além disso, foi verificado o valor preditivo da frequência semanal e do tempo de prática de meditação sobre os fatores do BES. A partir dos objetivos, foram levantadas as seguintes hipóteses: $\mathrm{H} 1$ ) os praticantes e não praticantes de meditação se diferenciarão quanto aos seus resultados na EBES, com os praticantes de meditação obtendo maiores escores em Satisfação com a Vida e Afetos Positivos, e menores em Afetos Negativos (Bach \& Guse, 2014; Galante et al., 2014; Goyal et al., 2014; Menezes \& Bizarro, 2015; Menezes \& Dell'Aglio, 2009; Menezes \& Dell'Aglio, 2010; Shonin et al., 2014; Tarrant et al., 2016); H2) quanto maior a frequência de prática de meditação por semana, maiores serão os escores em Satisfação com a Vida e Afetos Positivos, e menores em Afetos Negativos (Bussing et al., 2012; Galante, et al., 2014; Gaiswinkler et al., 2015; Menezes \& Dell'Aglio, 2010; Tarrant et al., 2016); e H3) quanto maior o período de tempo desde o início das práticas de meditação, isto é, anos de prática, maiores serão os escores em Satisfação com a Vida e Afetos Positivos, e menores em Afetos Negativos (Bussing et al., 2012; Galante, et al., 2014; Gaiswinkler et al., 2015; Tarrant et al., 2016).

\section{MÉTODO}

\section{PARTICIPANTES}

Débora Noemi Hernández, Gusta vo Henrique Martins
Participaram da presente pesquisa 303 sujeitos, ambos os sexos, sendo $78,5 \%$ do sexo feminino, com idades variando de 18 a 75 anos $(M=35,55 ; D P=13,30)$. A maioria dos participantes residiam na região Sudeste do Brasil (92,4\%). Dentre todos os participantes, $164(54,1 \%)$ declararam ser praticantes de meditação e 139 (45,9\%) declararam não praticar meditação. A média de idade dos participantes que praticavam meditação é de 39,19 anos (DP $=14,18)$, sendo $131(79,9 \%)$ do sexo feminino. Esses participantes se classificaram como praticantes de 21 tipos de meditações diferentes, sendo que a maioria praticava a Meditação dos Corações Gêmeos (51,8\%), Mindfulness $(12,8 \%)$ ou Yoga $(7,9 \%)$. Além disso, os praticantes de meditação informaram níveis de escolaridade de ensino médio incompleto $(0,6 \%)$, ensino médio completo $(9,1 \%)$, superior incompleto (21,3\%), superior completo (39,6\%) e com pós-graduação, como MBA, Especialização, Mestrado ou Doutorado (28,7\%). Os participantes que se identificaram como não praticantes de nenhum tipo de meditação, maioria do sexo feminino (77\%), apresentaram média de idade de 31,34 anos $(D P=10,81)$. Além disso, os níveis de escolaridade dos participantes foram ensino médio completo (8,6\%), superior incompleto $(37,4 \%)$, superior completo $(33,1 \%)$ e com pós-graduação, como MBA, Especialização, Mestrado ou Doutorado (20,9\%).

\section{INSTRUMENTOS}

\section{Questionário de identificação:}

No questionário, os participantes responderam a questões sobre idade, sexo, estado de residência e grau de escolaridade. Além disso, foram incluídas as seguintes perguntas: "considerando que meditar quer dizer manter a concentração em um som, objeto, momento, ou em si mesmo, você pratica algum tipo de meditação? $(1=$ não; 2 = sim)", "que tipo de meditação você pratica? (nome)", "em média, quantas vezes por semana você realiza esta prática? (utilize os números de 1 a 7 para identificar a quantidade de dias semanais)" e "aproximadamente há quanto tempo você realiza as práticas desta meditação? (anos ou meses)".

Escala de Bem-Estar Subjetivo - EBES (Albuquerque \& Tróccoli, 2004):

A escala avalia o BES por meio de 62 itens que representam os seguintes fatores: Afetos Positivos (21 itens), Afetos Negativos (26 itens) e Satisfação com a Vida (15 itens). 0 instrumento é respondido em escala Likert de 


\section{WL INTERACÃO EM
PSICOLOGIA}

5 pontos, sendo que nos fatores Afetos Positivos e Afetos Negativos, o sujeito deve responder à frase "ultimamente tenho me sentido..." para diversos sentimentos, em uma escala que varia de " 1 - nem um pouco" a "5 extremamente". Alguns exemplos para o fator Afetos Positivos na escala são: amável, ativo, alegre, disposto, seguro, produtivo e empolgado. Já alguns exemplos do fator Afetos Negativos são: agitado, angustiado, entediado, estressado, impaciente, nervoso e triste. Ao passo que, para o fator Satisfação com a Vida, a escala de resposta varia de "1 - discordo plenamente" a "5 - concordo plenamente", com itens como: "Avalio minha vida de forma positiva", "Mudaria meu passado se eu pudesse" e "Minhas condições de vida são muito boas". A precisão dos fatores para este estudo foram todos excelentes, com alfas de 0,93 (Satisfação com a Vida) e 0,96 (Afetos Positivos e Afetos Negativos).

\section{PROCEDIMENTOS}

0 projeto foi elaborado e encaminhado para avaliação do Comitê de Ética em Pesquisa da Universidade São Francisco. Após a aprovação (CAAE: 68323617.4.0000.5514), a coleta de dados ocorreu de forma presencial e online, ambos compostos pelo Termo de Consentimento Livre Esclarecido (TCLE), o questionário de identificação e a EBES. A coleta presencial ocorreu em um único momento, com participantes de um grupo de meditação, sendo realizada por uma estudante de Psicologia e pelo líder do grupo de meditação. Em paralelo, a coleta online foi realizada por meio do questionário informatizado na plataforma Google Formulários. Os convites, contendo o link para a pesquisa, foram enviados por e-mail e redes sociais. Ao acessarem o link da pesquisa, foram apresentados os objetivos, e somente após a anuência do TCLE, os participantes responderam aos instrumentos da pesquisa. O tempo médio de aplicação foi de aproximadamente 20 minutos por indivíduo.

\section{ANÁLISE DE DADOS}

As análises foram realizadas nos softwares SPSS
Débora Noemí Hernández, Gusta vo Henrique Martins

25 e MPlus 7.11. Inicialmente, foram realizadas análises descritivas para caracterização da amostra. Para verificar os objetivos deste estudo, foi testado um modelo preditivo por meio de uma Modelagem de Equações Estruturais (Structural Equation Modeling; SEM), com a prática de meditação, escolaridade, sexo e idade como variáveis independentes, e os três fatores do BES como variáveis dependentes (Satisfação com a Vida, Afetos Negativos e Afetos Positivos). Foram observadas diferenças entre os grupos de meditação em relação às variáveis escolaridade, sexo e idade. Portanto, optou-se pela inclusão dessas variáveis sociodemográficas no modelo testado, a fim de controlar os efeitos destas e tornar mais confiável a comparação dos grupos de meditação.

Em seguida, somente com a amostra das pessoas que praticavam meditação $(n=164)$, foram testados dois outros modelos preditivos, alterando-se apenas as variáveis independentes. No primeiro modelo, foi utilizada a variável frequência semanal como variável independente, e no segundo foi utilizada a variável tempo de prática. Em ambos os casos as variáveis dependentes foram os fatores do BES. $O$ ajuste do modelo foi verificado por meio dos seguintes índices de ajustes: razão entre quiquadrado e graus de liberdade $\left(\mathrm{X}^{2} / \mathrm{gl}<2\right)$, Confirmatory Fit Index (CFI; > 0,90), Tucker-Lewis Index (TLI; >0,90), Root Mean Square Error of Approximation (RMSEA; $<0,07$ ) (Brown, 2006).

\section{RESULTADOS}

A fim de se atingir os objetivos deste estudo, foi testado o modelo preditivo da prática de meditação sobre a Satisfação com a vida, Afetos Negativos e Afetos Positivos, sendo essa relação controlada pela escolaridade, sexo e idade dos participantes. 0 ajuste do modelo foi considerado aceitável $\left(X^{2} / g l=1,85 ; \mathrm{CFI}=0,93\right.$; $\mathrm{TLI}=0,93$; RMSEA $=0,05)$. As cargas fatoriais dos itens da EBES, na Análise Fatorial Confirmatória, foram em sua maioria acima de 0,40 , exceto o item " 46 - agitado", que apresentou carga fatorial de 0,27 no fator Afetos Negativos. A seguir, na Figura 1, é possível verificar os valores preditivos do modelo descrito anteriormente. 
Figura 1. Modelo preditivo da prática de meditação sobre o BES, controlada pela escolaridade, sexo e idade.

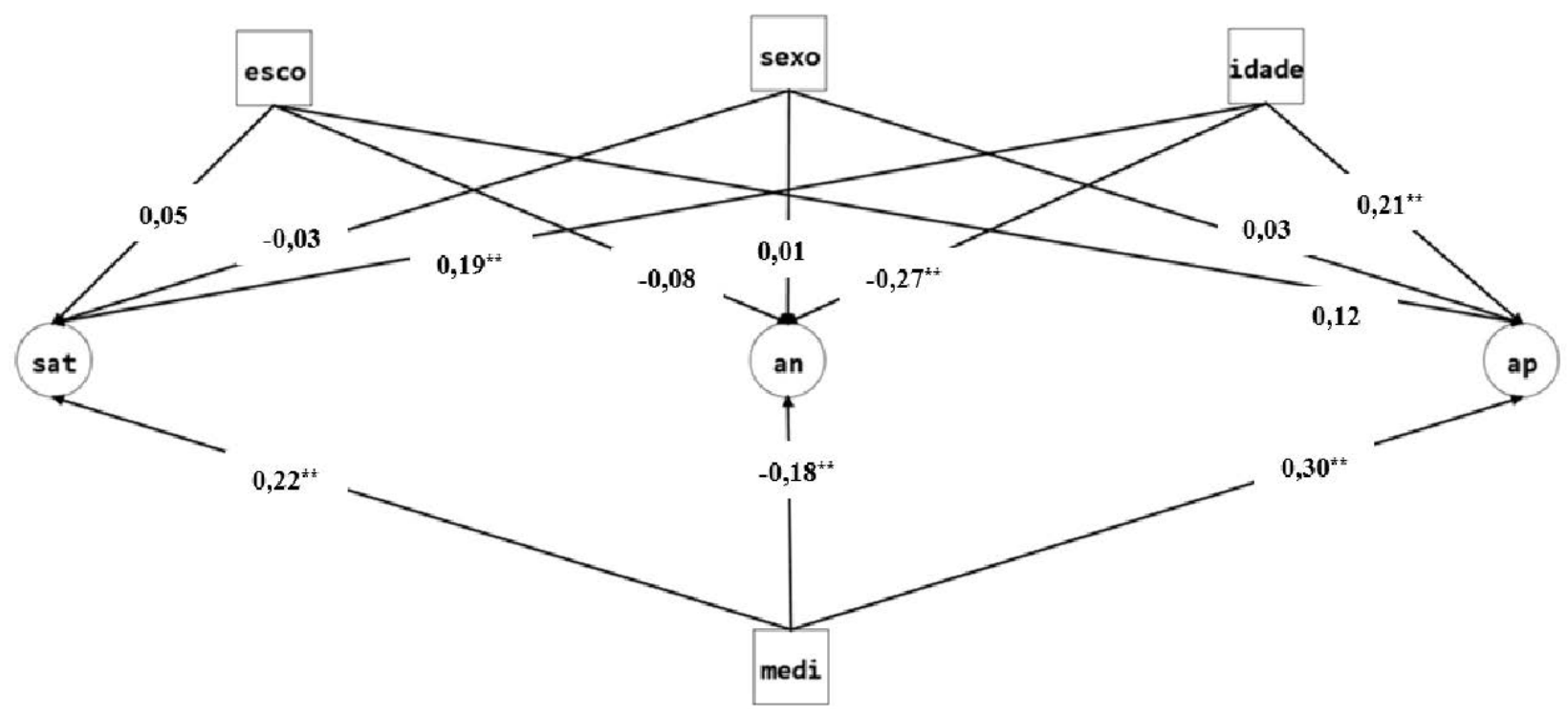

Nota. Os itens que representam os fatores da EBES foram suprimidos para a apresentação dos resultados. Variância explicada: Satisfação com a Vida $\left(r^{2}=0,12\right)$, Afeto Negativos $\left(r^{2}=0,16\right)$, Afetos Positivos $\left(r^{2}=0,20\right)$; esco = escolaridade; sat = Satisfação com a Vida; an = Afetos Negativos; ap = Afetos Positivos; medi $=$ prática de meditação; escolaridade ( 1 = ensino médio incompleto, 2 = ensino médio completo, 3 = ensino superior incompleto, 4 = ensino superior completo e 5 = pós-graduação como MBA, especialização, Mestrado ou Doutorado); sexo (1 = feminino e 2 = masculino); prática de meditação ( 1 = não e $2=\operatorname{sim})$.

$$
\star *: p<0,01 \text {. }
$$

Nota-se na Figura 1 que, dentre as variáveis sociodemográficas, apenas a idade foi uma preditora significativa dos três fatores do BES, sendo que quanto maior a idade dos indivíduos, maiores são seus índices de Satisfação com a Vida e de Afetos Positivos, e menores são os índices de Afetos Negativos. Por sua vez, quanto à variável prática de meditação, foi visto que esta também demonstrou ser preditora significativa dos três fatores do BES, sendo seus valores preditivos superiores aos da idade nos fatores Satisfação com a Vida e Afetos Positivos. Sendo assim, esse resultado indicou que as pessoas que meditam são mais satisfeitas com as suas vidas, e apresentam mais afetos positivos e menos afetos negativos, em comparação com aquelas que não praticam nenhum tipo de meditação, sendo essa relação independente da idade, sexo e escolaridade das pessoas.

As próximas análises foram realizadas somente com os participantes que declararam praticar meditação $(n=$ 164). A primeira delas foi realizada a partir da frequência semanal de prática de meditação dos participantes, com o intuito de explorar se essa especificidade dos praticantes de meditação pode explicar o BES. Para que houvesse uma distribuição similar entre as opções de resposta, algumas categorias foram agrupadas, sendo essa variável representada da seguinte forma: $1=$ uma vez por semana $(f=28), 2=$ duas vezes por semana $(f$ $=36), 3=$ três vezes por semana $(f=32), 4=$ quatro ou cinco vezes por semana $(f=32)$, e $5=$ seis ou sete vezes por semana $(f=36)$. Na Figura 2 , apresentada a seguir, 
Figura 2. Modelo preditivo da frequência semanal da prática de meditação sobre o BES.

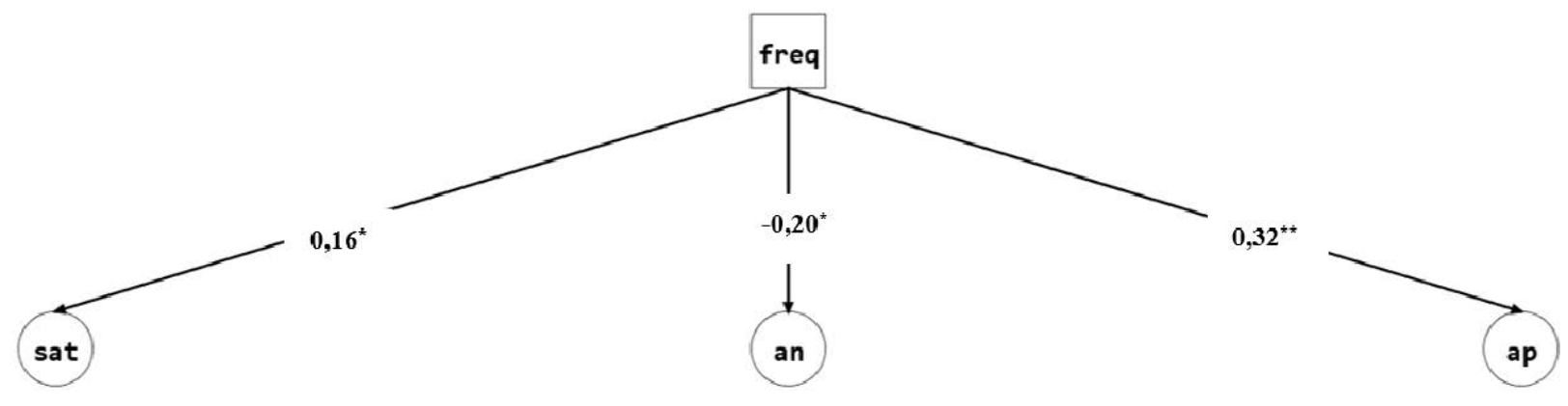

estão descritos os valores preditivos da frequência semanal sobre os fatores do BES.

Nota. Os itens que representam os fatores da EBES foram suprimidos para a apresentação dos resultados. Índices de ajuste: $\mathrm{X}^{2} / \mathrm{gl}=1,52 ; \mathrm{CFI}=0,92 ; \mathrm{TLI}=0,92$; RMSEA $=0,06$. Variância explicada: Satisfação com a Vida $\left(r^{2}=0,03\right)$, Afeto Negativos $\left(r^{2}=0,04\right)$, Afetos Positivos $\left(r^{2}\right.$ $=0,10)$; freq $=$ frequência semanal; sat $=$ Satisfação com a Vida; an = Afetos Negativos; ap = Afetos Positivos.

$$
*: p<0,05 ; * *: p<0,01 \text {. }
$$

Como é possível observar na Figura 2, a frequência semanal que os indivíduos praticavam meditação prediz positivamente a Satisfação com a Vida e os Afetos
Positivos, e negativamente os Afetos Negativos. Sendo assim, os resultados sugerem que há uma tendência a um maior BES por aqueles que praticam meditação várias vezes por semana, em comparação com aqueles que também praticam meditação, porém menos vezes por semana. Por fim, no último modelo preditivo testado, o qual será apresentado na Figura 3 , foi utilizada a variável tempo de prática de meditação. Buscando-se uma frequência mais próxima entre as opções, algumas respostas foram agrupadas, sendo essa variável representada da seguinte forma: $1=$ menos de um ano $(f=38), 2=$ de um ano e um mês a três anos $(f=39) ; 3=$ de quatro a nove anos $(f=46)$, e $4=$ mais de dez anos $(f$ $=41$ ).

Figura 3. Modelo preditivo do tempo de prática de meditação sobre o BES.

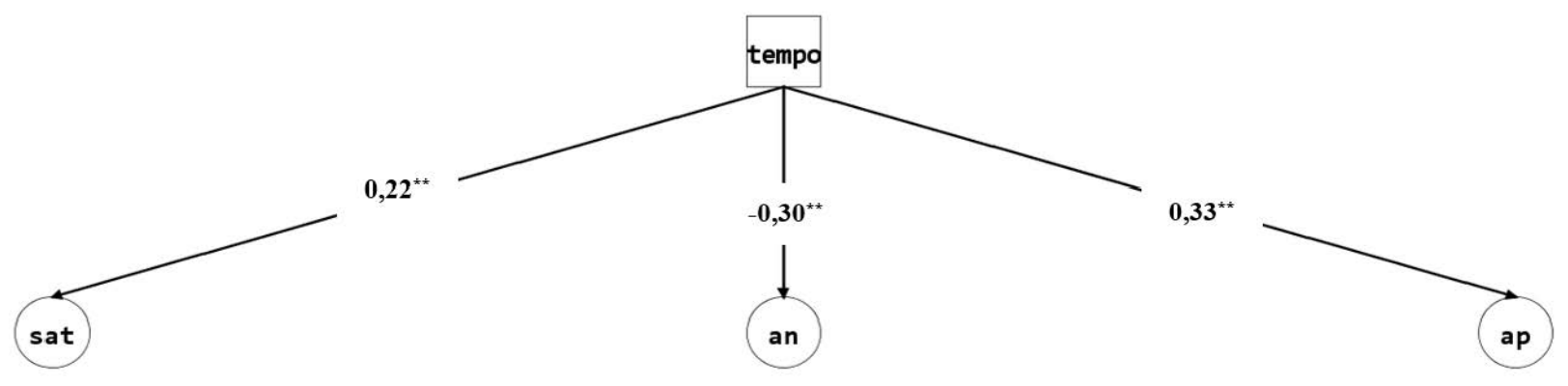

Nota. Os itens que representam os fatores da EBES foram suprimidos para a apresentação dos resultados. Índices de ajuste: $\mathrm{X}^{2} / \mathrm{gl}=1,52 ; \mathrm{CFI}=0,92 ; \mathrm{TLI}=0,92$; RMSEA $=0,06$. Variância explicada: Satisfação com a Vida $\left(r^{2}=0,05\right)$, Afeto Negativos $\left(r^{2}=0,09\right)$, Afetos Positivos $\left(r^{2}\right.$
$=0,11) ;$ tempo $=$ tempo de prática de meditação; sat $=$ Satisfação com a Vida; an = Afetos Negativos; ap = Afetos Positivos.

$$
\star *: p<0,01 .
$$


Débora Noemi Hernández, Gusta vo Henrique Martins

Constata-se na Figura 3 que a variável tempo de prática de meditação foi preditora significativa e positiva da Satisfação com a Vida e dos Afetos Positivos, e negativa dos Afetos Negativos. Esse resultado indica uma tendência de que pessoas que realizam as práticas de meditação há um longo tempo demonstram ter maiores níveis de BES, em comparação aos que iniciaram a prática há menos tempo.

\section{DISCUSSÃO}

O presente estudo buscou verificar a existência de diferenças no BES entre praticantes e não praticantes de meditação, controlando os efeitos da idade, sexo e escolaridade. Além disso, foi verificado o valor preditivo da frequência semanal e do tempo de prática de meditação sobre os fatores do BES. Nesse sentido, a partir dos resultados obtidos, as três hipóteses levantadas para este estudo foram corroboradas e serão descritas e discutidas a seguir.

A respeito das variáveis sociodemográficas testadas como preditoras do BES, foi visto que apenas a idade apresentou resultados significativos, sendo a idade preditora positiva do BES. Esse resultado é corroborado por outros estudos que também apontaram que pessoas com faixas etárias mais elevadas possuem mais Afetos Positivos e Satisfação com a Vida, e menos Afetos Negativos, em comparação com os mais jovens (Carochinho, 2018; Cunha et al., 2017; Otta $\&$ Fiquer, 2004). Por sua vez, o sexo e a escolaridade dos participantes dessa pesquisa demonstraram não predizerem significativamente os componentes do BES, contrastando outros estudos publicados anteriormente (Batz \& Tay, 2018; Cachioni et al., 2017; Freire \& Tavares, 2011; Noronha et al., 2016; Silva et al., 2010; Zanon \& Hutz, 2009). Ressalta-se que a inserção dessas variáveis sociodemográficas na análise teve o intuito de controlar seus possíveis efeitos na relação entre prática ou não de meditação e BES, uma vez que tais características sociodemográficas não foram perfeitamente simétricas entre os grupos de praticantes e não praticantes de meditação.

Ainda nesta primeira análise, notou-se que o grupo de pessoas que meditam apresentou maiores pontuações em Afetos Positivos e Satisfação com a Vida, e menores Afetos Negativos em comparação com quem não praticava meditação. Esses resultados podem estar relacionados à prática da meditação, considerada um treinamento da mente e desenvolvimento da concentração, por esta ser considerada uma ferramenta que promove a percepção positiva das situações e o aumento da calma emocional e mental, além da diminuição do estresse, raiva, irritabilidade e aflição emocional. Dessa forma, as práticas de meditação podem também contribuir para a Satisfação com a Vida e os Afetos Positivos, e para a diminuição da percepção dos Afetos Negativos (Mendoza, 2017; Shapiro et al., 2002). Outros pesquisadores também evidenciam a diminuição da ansiedade e aumento dos níveis de felicidade e autoestima, além de uma visão mais positiva no enfrentamento de dificuldades por parte dos praticantes de meditação (Balasubramaniam, et al., 2013; Forfylow, 2011; Menezes \& Dell'Aglio, 2009; Tarrant et al., 2016).

Outras pesquisas também identificaram efeitos positivos das práticas de meditação nos níveis do BES (Bach \& Guse, 2014; Galante, et al., 2014; Goyal et al., 2014; Menezes \& Bizarro, 2015; Menezes \& Dell'Aglio, 2009; Menezes \& Dell'Aglio, 2010; Shonin et al., 2014; Tarrant et al., 2016). Sendo assim, esses resultados indicam que praticantes de meditação experimentam mais sentimentos de prazer, como o entusiasmo, e menos estados emocionais de angústia e desconforto despertados pelo estímulo, e o julgamento sobre as suas próprias vidas tende a ser mais positivo (Albuquerque \& Tróccoli, 2004; Diener, 2000; Emmons, 1986; Shin \& Johnson, 1978; Watson et al., 1988). Portanto, os resultados do presente estudo corroboram a $\mathrm{H} 1$, cuja hipótese era que o grupo que praticava meditação apresentaria pontuações maiores de BES em comparação com os não praticantes.

Além disso, foram realizados outros dois modelos preditivos, nos quais foi constatado que tanto a frequência semanal como os anos de prática de meditação foram preditores positivos do BES. Com isso, observou-se índices mais elevados de Afetos Positivos e Satisfação com a Vida, e índices inferiores de Afetos Negativos, tanto para pessoas que praticam mais vezes por semana como para aqueles que realizam as práticas há mais anos. Esses resultados podem evidenciar que a meditação possui efeitos positivos no BES do sujeito, que podem ser alcançados de forma mais efetiva com a prática constante e ao longo do tempo.

Alguns autores sugerem que os efeitos temporais das práticas de meditação não se devam apenas à meditação propriamente dita, mas também à compreensão de que a maioria das meditações encontra-se acompanhada de uma filosofia de vida. Essa filosofia então é assumida progressivamente pelo sujeito no seu cotidiano conforme este se envolve nas práticas de meditação, a qual afetaria 
a maneira que vivencia os afetos para cada estímulo experienciado (Bussing et al., 2012; Galante, et al., 2014; Gaiswinkler et al., 2015; Menezes \& Dell'Aglio, 2010; Tarrant et al., 2016). No estudo de Menezes e Dell'Aglio (2010), as autoras identificaram uma relação positiva entre 0 número de meses e a frequência semanal da prática de meditação e o bem-estar psicológico. Especificamente em relação aos afetos, os autores Galante et al. (2014) observaram em sua pesquisa que os Afetos Positivos aumentaram progressivamente conforme a prática de meditação era utilizada. Dessa forma, a H2 e H3 também foram corroboradas a partir dos resultados deste estudo.

Perante os resultados apresentados, entende-se que existem evidências que possibilitam a compreensão da relação positiva entre as práticas de meditação e o BES dos sujeitos. Entende-se que devido à aprovação de práticas complementares no atendimento do Sistema Único de Saúde (SUS), perante a Portaria N ${ }^{\circ} 145 / 2017$, existe a necessidade atual de estudos científicos nacionais que procurem compreender mais profundamente os efeitos das mesmas. Nesse sentido, o presente trabalho pôde contribuir com a investigação dos efeitos de da prática de meditação sobre o BES no contexto brasileiro, ampliando o conhecimento científico sobre o assunto que é escasso no país (Menezes \& Bizarro, 2015; Menezes \& Dell'Aglio, 2009; Menezes \& Dell'Aglio, 2010).

Contudo algumas limitações necessitam ser relatadas. Os participantes deste estudo eram praticantes de diversos tipos de meditação, com destaque para a Meditação dos Corações Gêmeos, que representou mais da metade da amostra, podendo este ser um viés nos resultados obtidos. Sugere-se, portanto, novas pesquisas nacionais que busquem avaliar os efeitos de cada tipo de meditação sobre o BES. Assim como colocado pelas autoras Menezes e Dell'Aglio (2009), existe a possibilidade de cada técnica de meditação ter um efeito diferente no BES dos indivíduos. Além disso, não foi controlado neste estudo o momento que os participantes meditaram, se foi antes ou depois de responder à pesquisa. Nesse sentido, sugere-se que estudos futuros avaliem o BES em um grupo imediatamente após a pessoa ter meditado, e em outro grupo após passadas pelo menos 24 horas da última meditação, a fim de verificar possíveis diferenças no BES dessas pessoas.

Embora as análises deste estudo tenham sido conduzidas com um método robusto para testar modelos preditivos (SEM), não é possível atestar causalidade, mas sim, relação entre meditação e BES. Dessa maneira, sugerem-se que novos estudos sejam realizados com delineamentos experimentais ou quase-experimentais, para que sejam testados os efeitos da meditação sobre o BES com maior robustez metodológica. Por fim, evidencia-se a meditação como uma possibilidade de prática integrativa e complementar para a promoção do BES no contexto brasileiro, dada a relação positiva entre praticantes de meditação e BES constatada neste estudo.

\section{DECLARAÇÃO DE CONTRIBUIÇÃO DOS AUTORES:}

Certificamos que todos os autores participaram suficientemente do trabalho para tornar pública sua responsabilidade pelo conteúdo. A contribuição de cada autor pode ser atribuída como se segue:

DNH foi responsável pela administração do projeto, conceitualização, investigação e tabulação dos dados. GHM foi responsável pela metodologia, análise formal dos dados e visualização. DNH e GHM foram responsáveis pela validação e redação final (revisão e edição).

\section{DECLARAÇÃO DE CONFLITOS DE INTERESSE:}

Os autores declaram que não há conflitos de interesse neste artigo.

\section{REFERÊNCIAS}

Albuquerque, A. S. \&Tróccoli,B. T. (2004). Desenvolvimento de Uma Escala de Bem-Estar Subjetivo. Psicologia: Teoria e Pesquisa, 20(2),153-164. https://doi. org/10.1590/S0102-37722004000200008

Bach, J. M. \& Guse, T. (2014). The effect of contemplation and meditation on 'great compassion' on the psychological well-being of adolescents. The Journal of Positive Psychology, 10(4). https://doi.org/10.1080/ 17439760.2014.965268

Balasubramaniam, M., Telles, S., and Doraiswamy, P. M. (2013). Yoga on our minds: A systematic review of yoga for neuropsychiatric disorders. Frontiers in Psychiatry, 3(117). https://doi.org/10.3389/fpsyt.2012.00117

Baptista, M. N., Hauck Filho, N. \& Cardoso, C. (2016). Depressão e bem-estar subjetivo em crianças e adolescentes: teste de modelos teóricos. Psico, 47(4), 259-267. https://doi.org/10.15448/19808623.2016.4.23012

Batz, C., \& Tay, L. (2018). Gender differences in subjective well-being. Em E. Diener, S. Oishi, \& L. Tay (Eds.), Handbook of well-being. DEF Publishers.

Brown, T. A. (2006). Confirmatory Factor Analysis for Applied Research. Guilford Publications. 
Büssing, A., Hedtstück, A., Khalsa, S. B. S., Ostermann, T., \& Heusser, P. (2012). Development of specific aspects of spirituality during a 6-month intensive yoga practice. Evidence-Based Complementary and Alternative Medicine, 2012(special issue), 1-7. https:// doi.org/10.1155/2012/981523

Cachioni, M., Delfino, L. L., Yassuda, M. S., Batistoni, S. S. T., Melo, R. C., \& Domingues, M. A. R. C. (2017). Bemestar subjetivo e psicológico de idosos participantes de uma Universidade Aberta à Terceira Idade. Revista Brasileira de Geriatria e Gerontologia, 20(3). 340-352. https://doi.org/10.1590/1981-22562017020.160179

Carochinho, J. A. (2018). Adaptação e Validação para a Língua Portuguesa de um Conjunto de Escalas de Bem-estar. Revista Iberoamericana de Diagnóstico y Evaluación-e Avaliação Psicológica, 1(46), 37-50. https://doi.org/10.21865/RIDEP46.1.03

Cunha, M., Duarte, J., Sandré, S., Sequeira, C., CastroMolina, F. J., Mota, M., Pina, F., Coelho, M., Cunha, A., Figueiredo, A., Martins, A., Correia, B., Monteiro, D., Moreira, F., Silva, M., \& Freitas, S. (2017). Bem-estar em estudantes do ensino superior. Millenium - Journal of Education, Technologies and Health, 2(ed espec $\left.n^{\circ} 2\right)$, 21-38. https://doi.org/10.29352/mill0202e.02

Diener, E. (2000). Subjective well-being: The science of happiness and a proposal for a national index. American Psychologist, 55(1), 34-43.

Diener, E., Oishi, S. \& Lucas, R. E. (2003). Personality, culture and subjective well-being: Emotional and cognitive evaluations of life. Annual Review of Psychology, 54, 403-425. https://doi.org/10.1146/ annurev.psych.54.101601.145056

Duckworth, A. L., Steen, T. A., \& Seligman, M. E. P. (2005). Positive psychology in clinical practice. Annual Review of Clinical Psychology, 1, 629-651. https://doi. org/10.1146/annurev.clinpsy.1.102803.144154

Emmons, R. A. (1986). Personal strivings: An approach to personality and subjective well-being. Journal of Personality and Social Psychology, 51(5), 1058-1068. https://doi.org/10.1037/0022-3514.51.5.1058

Forfylow, A. L. (2011). Integrating yoga with psychotherapy: a complementary treatment for anxiety and depression. Canadian Journal of Counselling and Psychotherapy, 45(2), 132-150.

Freire, T. \& Tavares, D. (2011). Influência da autoestima, da regulação emocional e do gênero no bem-estar subjetivo e psicológico de adolescentes. Revista de Psiquiatria Clínica, 38(5), 184-188. https://doi. org/10.1590/S0101-60832011000500003
Gaiswinker, L., Unterrainer, H. F., Fink, A., \& Kapfhammer, H. P. (2015). Die Beziehung zwischen yoga immersion, psychologischem wohlbefinden und psychiatrischer symptomatik [The relationship between yoga immersion, psychological well-being and psychiatric symptoms]. Neuropsychiatrie, 29, 29-35. https://doi. org/10.1007/s40211-015-0139-9

Galante, J., Galante, I., Bekkers, M. J., \& Gallacher, J. (2014). Effect of kindness-based meditation on health and well-being: A systematic review and meta-analysis. Journal of Consulting and Clinical Psychology, 82(6), 1101-1114. https://doi.org/10.1037/a0037249

Goyal, M., Singh, S., Sibinga, E. M. S., Gould, N. F., RowlandSeymour, A., Sharma, R., Berger, Z., Sleicher, D., Maron, D. D., Shihab, H. M., Ranasinghe, P. D., Linn, S., Saha, S., Bass, E. B., \& Haythornthwaite, J. A. (2014). Meditation Programs for Psychological Stress and Well-being. JAMA Internal Medicine, 174(3), 357-368. https://doi. org/10.1001/jamainternmed.2013.13018

Keyes, C. L. M., Shmotkin, D. \& Ryff, C. D. (2002). Optimizing well being: The empirical encounter of two traditions. Journal of Personality and Social Psychology, 82(6), 1007-1022. https://doi.org/10.1037//00223514.82.6.1007

Larsen, R. J., Diener, E. \& Emmons, R. A. (1985). An evaluation of subjective well-being measures. Social Indicators Research, 17, 1-17. https://doi.org/10.1007/ BF00354108

Lyubomirsky, S., King, L., \& Diener, E. (2005). The benefits of frequent positive affect: Does happiness lead to success? Psychological Bulletin, 131(6), 803-855. https://doi.org/10.1037/0033-2909.131.6.803

Mendoza, G. J. M. D. (2017). Better Person Mendset. Back Card Books.

Menezes, C. B., \& Bizarro, L. (2015). Effects of a brief meditation training on negative affect, trait anxiety and concentrated attention. Paidéia, 25(62), 393-401. https://doi.org/10.1590/1982-43272562201513

Menezes, C. B., \& Dell'Aglio, D. D. (2009). Por que meditar? A experiência subjetiva da prática de meditação. Psicologia em Estudo, 14(3), 565-573. https://doi. org/10.1590/S1413-73722009000300018

Menezes, C. B., \& Dell'Aglio, D. D. (2010). The relationship between the practice of sitting and silent meditation and psychological well-being and the effects of personality traits. Interamerican Journal of Psychology, 44(1), 140-149.

Ministério da Saúde. (2019). Política Nacional de Práticas Integrativas e Complementares em Saúde. http://dab. saude.gov.br/portaldab/pnpic.php 
Moebus, R. L. N., \&Merhy,E. E. (2017). Genealogia dapolítica nacional de práticas integrativas e complementares. Saúde em Redes, 3(2), 145-152. https://doi. org/10.18310/2446-4813.2017v3n2p145-152

Nikhra, M. (2016). A study on the effect of yoga \& pranic healing on psychological disorder. International Journal of Science and Consciousness, 2(1), 41-44.

Noronha, A. P. N., Lamas, K. C. A., \& Barros, M. V. C. (2016). Afetos e personalidade: Suas relações em estudantes universitários. Psicologia Teoria e Prática, 18(2), 7588. https://doi.org/10.15348/1980-6906/psicologia. v18n2p75-88

Otta, E. \& Fiquer, J. T. (2004). Bem-estar subjetivo e regulação de emoções. Psicologia em Revista, 10(15), 144-149.

Pires, J. G., Nunes, M. F. O., \& Nunes, C. H. S. D. S. (2015). Instrumentos baseados em psicología positiva no Brasil: Uma revisão sistemática. Psico-USF, 20(2), 287295. https://doi.org/10.1590/1413-82712015200209

Portaria $\mathrm{N}^{\circ} 145$ de 11 de janeiro de 2017. Altera procedimentos na Tabela de Procedimentos, Medicamentos, Órteses, Próteses e Materiais Especiais do SUS para atendimento na Atenção Básica. http://www.in.gov.br/materia/-/asset_publisher/ Kujrw0TZC2Mb/content/id/20581305/do1-2017-0113-portaria-n-145-de-11-de-janeiro-de-2017-20581242

Pureza, J. R., Kuhn, C. H. C., Castro, E. K., \& Lisboa, C. S. M. (2012). Psicologia positiva no Brasil: uma revisão sistemática da literatura. Revista Brasileira de Terapias Cognitivas, 8(2), 109-117. https://doi. org/10.5935/1808-5687.20120016

Ribeiro, W. R. B., Gindom, S. M. G. \& Pereira, C. R. (2018). Personalidade e bem-estar subjetivo de trabalhadores: moderação da regulação emocional. Arquivos Brasileiros de Psicologia [online], 70(1), 239259. Recuperado de http://pepsic.bvsalud.org/pdf/ arbp/v70n1/17.pdf

Scorsolini-Comin, F., Fontaine, A. M. G. V., Barroso, A. M. \& Santos, M. A. (2016). Fatores associados ao bemestar subjetivo em pessoas casadas e solteiras. Estudos de Psicologia, 33(2), 313-324. https://doi. org/10.1590/1982-02752016000200013

Scorsolini-Comin, F., Fontaine, A. M. G. V., \& Santos, M. A. (2015). Conjugalidade dos pais: percepções de indivíduos casados e solteiros. Avaliação Psicológica, 14(2), 223-231. https://doi.org/10.15689/ ap.2015.1402.07

Seligman, M. E. P. (2011). Florescer: Uma nova compreensão sobre a natureza da felicidade e do bemestar. Objetiva.

Shapiro, S. L., Schwartz, G. E. R., \& Santerre, C. (2002). Meditation and positive psychology. Em C. R. Snyder \& S. J. Lopez (Eds.), The handobook of positive psychology (632-645). Oxford university press.
Shin, D. C. \& Johnson, D. M. (1978). Avowed happiness as an overall assessment of the quality of life. Social Indicators Research, 5, 475-492. https://doi. org/10.1007/BF00352944

Shonin, E., Van Gordon, W. \& Griffiths, M. D. (2014). Meditation Awareness Training (MAT) for improved psychological well-being: A qualitative examination of participant experiences. Journal of Religion and Health, 53(3), 849-863. https://doi.org/10.1007/ s10943-013-9679-0

Silva, A. B., Matos, M. G. \& Diniz, J. A. (2010). Idade, Género e Bem-Estar Subjectivo nos Adolescentes. Revista Portuguesa de Pedagogia, 444(2), 39-61.

Snyder, C. R. \& Lopez, S. J. (2009). Psicologia positiva: Uma abordagem científica e prática das qualidades humanas. Artmed.

Tarrant, J., Raines, N., Blinne, W. \& Perez, N. (2016). sLORETA and psychological functioning impacts from meditation on twin hearts: Differences between novice and experienced. Integrative Medicine, 15(1). https://search.proquest.com/open view/587ba7de162ca1854e9c367587fd778a/1?pqorigsite $=$ gscholar $\& \mathrm{cbl}=736337$

Walsh, L. C., Boehm, J. K., \& Lyubomirsky, S. (2018). Does happiness promote career success? Revisiting the evidence. Journal of Career Assessment, 26(2), 199219. https://doi.org/10.1177/1069072717751441

Watson, D., Clark, L. A., \& Tellegen. A. (1988). Development and validation of brief measures of positve and negative affect: The PANAS Scales. Journal of Personality and Social Pychology, 54(6), 1063-1070. https://doi.org/10.1037/0022-3514.54.6.1063

World Health Organization - WHO. (2013). Estrategia de la OMS sobre medicina tradicional 20142023. https://apps.who.int/iris/bitstream/ handle/10665/95008/97892435060908_spa. ionid=C192292FEF8E3942FDF45A9D0EB8997E? sequence $=1$

Zanon, C., \& Hutz, C. S. (2009). Relações entre bem-estar subjetivo, neuroticismo, ruminação, reflexão e sexo. Gerais: Revista Interinstitucional de Psicologia [online], 2(2), 118-127. http:// pepsic.bvsalud.org/scielo.php?script =sci_ arttext\&pid=S1983-82202009000200007

Data de submissão: 12/02/2019 Primeira decisão editorial: 09/05/2019 Aceite em 31/07/2019 